\title{
EFFICACY OF TWO DIFFERENT TREATMENT MODALITIES ON MASKING WHITE SPOT LESIONS IN CHILDREN WITH MOLAR INCISOR HYPO-MINERALIZATION
}

\author{
Ghada A. ElBaz * and Shaimaa M. Mahfouz **
}

\begin{abstract}
Purpose: This study aimed to assess and compare the effectiveness of resin infiltration and fluoride varnish in masking white spot lesions of central incisors in children with molar incisor hypo-mineralization (MIH) clinically and radio-graphically.

Methods: Twenty children aged from 9 to 14 years with bilateral maxillary central incisors with MIH, according to Judgment criteria of European Academy of Pediatric Dentistry (EAPD), were included in this study. Children were divided into two groups, group I was treated with resin infiltration (ICON) while group II was treated with fluoride varnish (Fluoro- protector). All patients received pre-operative, immediately after treatment, 1 week and 1 month after treatment a digital standardized photograph and peri-apical radiography. All photographs and peri-apical radiographs were analyzed to calculate the color and gray level (GL) differences between the sound enamel and white spot lesions.
\end{abstract}

Results: A comparison between the two groups showed a statistically significant lower color difference mean between the sound and treated white spots enamel in group I than in group II in all evaluation periods. Moreover, a comparison between the GL mean values of both groups showed a statistically significant higher GL mean values after resin infiltrations in group I than in group II after fluoride application in all evaluation periods.

Conclusion: Resin infiltration (ICON) is dramatically better than fluoride varnish in the masking of white spot lesions in children with (MIH). Resin infiltration technique allows a minimally invasive treatment in a single appointment making it beneficial for school aged patients.

KEYWORDS: White spot-fluoride- resin infiltration-children.

* Assistant Professor of Pediatric and Preventive Dentistry and Dental Public Health, Faculty of Dentistry, Suez Canal University.

** Lecturer of Pediatric and Preventive Dentistry and Dental Public Health, Faculty of Dentistry, Suez Canal University. 


\section{INTRODUCTION}

Molar incisor hypo-mineralization (MIH) is a frequently noticed developmental defect of dental enamel that involves hypo-mineralization of 1 to 4 permanent first molars (FPM) with or without permanent incisors. The qualitative structure enamel defects occur mainly due to systemic causes 1,2. MIH may be due to environmental factors such as chronic or acute illness during last gestational trimester and first 3 years of childhood more precisely during the period in which the crowns of the FPMs and incisors are mineralized ${ }^{3}$. MIH clinically appears as a well demarcated discrete opaque lesions ranging from white to yellow-brown color of normal thickness with a smooth surface, and has a distinct boundary adjacent to normal enamel ${ }^{3,4}$. Based on previous research on $\mathrm{MIH}$, it has been shown that $71.6 \%$ of affected children may have permanent incisors as well as FPM involved ${ }^{5}$.The children with MIH suffer from aesthetic and psychological problems that need to be treated as early as possible ${ }^{6}$. Many studies reported that topical application of fluoride or casein phosphopeptide amorphous calcium phosphate (CPP-ACP) is successfully re-mineralizing the de-mineralized enamel surface but their aesthetic effect is limited because re-mineralization is limited to the lesion surface ${ }^{7-10}$. Recently, correction of enamel lesions by resin infiltration to achieve better enamel color and translucency seems to be promising approaches for the noninvasive treatment of white spot lesions. Infiltration of low viscosity resin into subsurface enamel layer has been used for decreasing enamel porosity and for obstructing minerals and acid pathway out of enamel surface, which arrest enamel surface demineralization ${ }^{11-13}$.Therefore, the aim of the present study was to assess and compare the effectiveness of resin infiltration and fluoride varnish in masking white spot enamel lesions of permanent central incisors in children with MIH clinically and radio-graphically.

\section{MATERIALS AND METHODS}

Twenty apparently healthy and cooperative children with bilateral maxillary permanent central incisors with MIH were included in this study. Their age ranged from 9 to 14 years and they were selected from Outpatient Clinic, Pedodontic Department, Faculty of Dentistry, Suez Canal University. The protocol of the research project has been revised and approved by the scientific ethical committee of Pedodontic Department, Faculty of Dentistry, Suez Canal University. A brief history and clinical examination were recorded. Hypo-mineralization area was assessed and identified visually as an abnormality in the translucency of the enamel and also denominated as opacity of the enamel according to Judgment criteria of European Academy of Pediatric Dentistry (EAPD) ${ }^{14}$. All patients had not received any previous treatment in both maxillary permanent central incisors. Treatment plan was explained to the parent and a written informed consent was obtained before the clinical procedure. Teeth included in this study were being cleaned with a rubber cups and prophylaxis paste. Each patient received pre-operative digital photography and digital periapical radiography. Children were divided into two groups of 10 patients each as follow: group I ( $\mathrm{n}=20$ maxillary central incisors) was treated with resin infiltration (ICON_ DMG) and group II ( $\mathrm{n}=20$ maxillary central incisors) was treated with Fluoro- protector (difluorsilane 1000 ppmF, Involar- Vivadent, Schaan, Liechtentein). The resin infiltration and fluoride varnish were applied to the teeth according to manufacturer's instructions.

Group I: Each patient received resin infiltration in two maxillary permanent central incisors. The teeth must be cleaned with a pumice or non fluoridated prophylaxis paste before the infiltration procedure. During the infiltration procedure, the eye glasses should be wore for protection also rubber dam was applied. The surface layer of the incisors was etched 
by the application of $15 \%$ hydrochloric acid gel in a circular motion (ICON_-Etch; DMG, Hamburg, Germany) for 2 minutes to expose the layer of lesion body and finally rinsed for 30 seconds with water spray and dried. The lesions were desiccated using $100 \%$ ethanol (ICON_-Dry; DMG) for 30 seconds followed by air dryness. An infiltrant resin (ICON_-Infiltrant; DMG) was applied to the surface and allowed to penetrate inside for 3 minutes with the operator light turned away. Excessive material was wiped off using a cotton roll from the surface and with a dental floss from the proximal surfaces before light curing for 40 seconds. The process was then repeated with the icon infiltrant being placed for one more minute then each tooth was cured for a further 40 seconds. Finally, the roughened enamel surface was polished using a composite resin polishing discs (Sof-lex disk; 3M ESPE, Saint Paul, MN, USA).

Group II: Each patient received a fluoride varnish application in two maxillary permanent central incisors. Thoroughly clean the tooth surfaces. Adequate isolation was performed by cotton roll and high suction. Fluoride varnish was applied by special applicator brush on a proper dried tooth surface. Generally, Fluor Protector is applied every six months according to manufacturer's instructions.

Standardized clinical digital photographs and digital radiographs were taken before treatment (P0\&R0) served as a baseline record for successive films, immediately after treatment (P1\&R1), one week (P2\&R2) and one month (P3\&R3) after treatment for both groups.

\section{I) Standardized clinical photography}

Standardized clinical photographs were taken by a camera, Nikon D3200, Thailand, lens, Nikkor 1855mm AF-S VR 1:3.5-5.6 G II , Thailand . A tripod was used to fix the camera at the same distance from the patient also; the dental unit was stabilized at rest position to standardize the photographic conditions.
The camera settings were $1 / 200$, F29, ISO 600, and Auto white balance. The flash was set on manual mode with power $1 / 16$ with no compensation. All photographs were taken by one photographer according to a standardized protocol.

\section{Evaluation of color changes}

The sound enamel and white spot lesions were outlined on the screen, and the colors of the sound enamel and white spot lesions were measured using an image analyzing photoshop program. The color of the teeth in the same site was analyzed by transferring the outlined layer of the P0 photograph to the P1, P2 and P3 photographs. The difference in color between the sound enamel and white spot lesions at each time was calculated and measured by digital analyzing photography program.

\section{II) Standardized digital radiography}

Standardized intraoral periapical digital radiographs were taken before treatment (R0), immediately after treatment (R1), one week (R2) and one month (R3) after treatment for each treated tooth using a long cone paralleling technique for standardization. An individual silicon bite stent and a film holder device, in which the X-ray tubefitted were used in order to achieve standardization. The sensor was placed horizontally in the holder for the central incisors region. The sensor was exposed to the dental $\mathrm{x}$-ray machine (Fona XCDItaly) at 100-240 V, 50-60 HZ , $6 \mathrm{~A}$ and $690 \mathrm{VA}$ for 0,06 seconds with $\mathrm{x}$-ray perpendicular to sensor. The exposure parameters were fixed for all patients. After exposure was terminated, the readout started automatically and the image was displayed gradually on a computer screen and the image was stored automatic. Baseline radiographs (R0) were compared with (R1), (R2) and (R3) radiographs for detection of changes in crown radio-density. Quantitative information regarding radio-density changes at crown areas was obtained by the use of a computerized image analysis program using 
Digora (2.5) image analysis software system. The optical density of each image element (pixel) was quantified and converted into gray levels between 0 and 255 ( 0 for black regions and 255 for white) by using an image processor (Digora) based on windows system.

\section{Statistical analysis}

The results were recorded, tabulated, and statistically analyzed using SPSS version 16 computer analysis software for statistical analysis. Data were expressed as mean \pm standard deviations of the parameters evaluated. Paired sample $t$ test was used to test the significance of the difference of intra group measurement, while independent sample t test was used to test the significance of the difference of inter group measurement. The significance level was set at $\mathrm{P}<0.05$.

\section{RESULTS}

\section{I) Color change results}

In group I, the mean color difference between the sound and white spots enamel was significantly decreased after immediate treatment, one week and one month after resin infiltration treatment from $204.99,194.83$ to 194.38 while in group II, the mean color difference was in-significantly decreased after immediate treatment, one week and one month after fluoride varnish application from 200.17, 198.44 to 198.4. On the other hand, a comparison between group I and group II showed a statistically significant lower mean color difference in group I than in group II in all evaluation periods. (Figures $1,2,3,4)($ Table 1$)$

\section{II) Gray level results}

Comparison of the gray level (GL) means of images taken before and after resin infiltrations or fluoride application showed that, for group I there was a significant improvement in radio-density from base line to one month after resin infiltration treatment from $83.98 \pm 0.9$ to $110.13 \pm 0.93$, while in group II there was in-significant improvement from base line to one month after fluoride varnish application ( $77.65 \pm 0.8$ to $78.55 \pm 1.33$ ). In addition, a comparison between the GL mean values of the group I and group II showed a statistically significant higher GL mean value in the group I than in group II in all evaluation periods. (Figure 5) (Table 2)

TABLE (1) Comparisons between the color difference results for both treated groups at different evaluation periods

\begin{tabular}{|c|c|c|c|c|}
\hline \multirow{2}{*}{ Color difference } & \multicolumn{2}{|c|}{ Treatment modalities } & \multicolumn{2}{c|}{} \\
\cline { 2 - 5 } & Resin infiltration & Fluoride varnish & Difference & P value \\
\hline Baseline (P0) & $204.99 \pm 13.85$ & $200.17 \pm 16$ & $4.82 \pm 10.17$ & $>0.05$ \\
\hline Immediate after treatment (P1) & $194.63 \pm 4.2$ & $198.93 \pm 2.9$ & $4.3 \pm 2.1$ & $<0.05^{*}$ \\
\hline 1 week (P2) & $194.83 \pm 4.7$ & $198.44 \pm 1.9$ & $4.61 \pm 3.7$ & $<0.05^{*}$ \\
\hline 1 month (P3) & $194.38 \pm 5.0$ & $198.4 \pm 2.1$ & $4.02 \pm 2.9$ & $<0.05^{*}$ \\
\hline $\begin{array}{c}\text { Baseline versus immediate, } \\
1 \text { week, and 1 month }\end{array}$ & $<0.05^{*}$ & $>0.05$ & & \\
\hline
\end{tabular}


TABLE (2) Comparisons between the gray level results for both treated groups at different evaluation periods

\begin{tabular}{|c|c|c|c|c|}
\hline \multirow{2}{*}{ Gray level } & \multicolumn{2}{|c|}{ Treatment modalities } & \multicolumn{2}{|c|}{} \\
\cline { 2 - 5 } & Resin infiltration & Fluoride varnish & Difference & P-value \\
\hline Baseline (R0) & $83.98 \pm 0.9$ & $77.65 \pm 0.8$ & $6.33 \pm 0.17$ & $>0.05$ \\
\hline Immediate after treatment (R1) & $110.83 \pm 0.35$ & $78.23 \pm 0.91$ & $32.6 \pm 0.98$ & $<0.05^{*}$ \\
\hline 1 week (R2) & $110.65 \pm 1.54$ & $78.83 \pm 1.5$ & $31.82 \pm 0.49$ & $<0.05^{*}$ \\
\hline 1 month (R3) & $110.13 \pm 0.93$ & $78.55 \pm 1.33$ & $31.58 \pm 0.69$ & $<0.05^{*}$ \\
\hline $\begin{array}{c}\text { Baseline versus immediate, 1 week, and } \\
1 \text { month }\end{array}$ & $<0.05^{*}$ & $>0.05$ & & \\
\hline
\end{tabular}

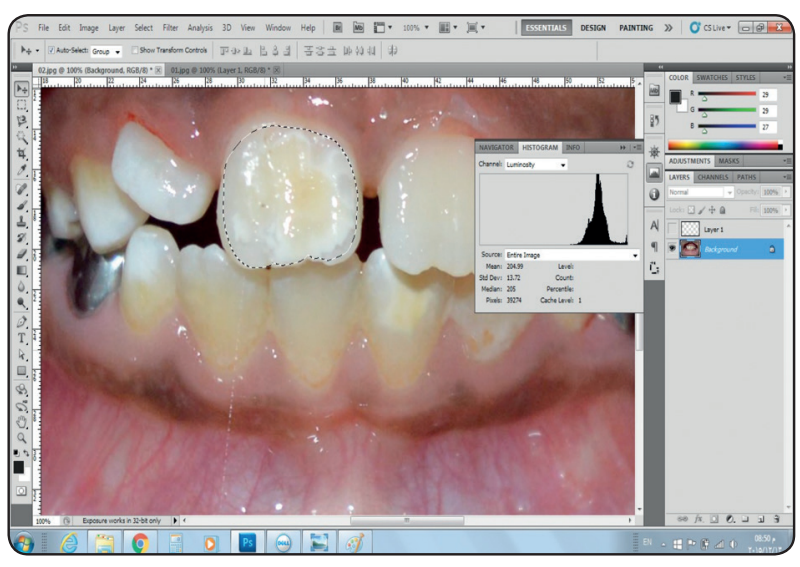

Fig. (1) Digital photography before treatment with resin infiltration $(\mathrm{P} 0)$

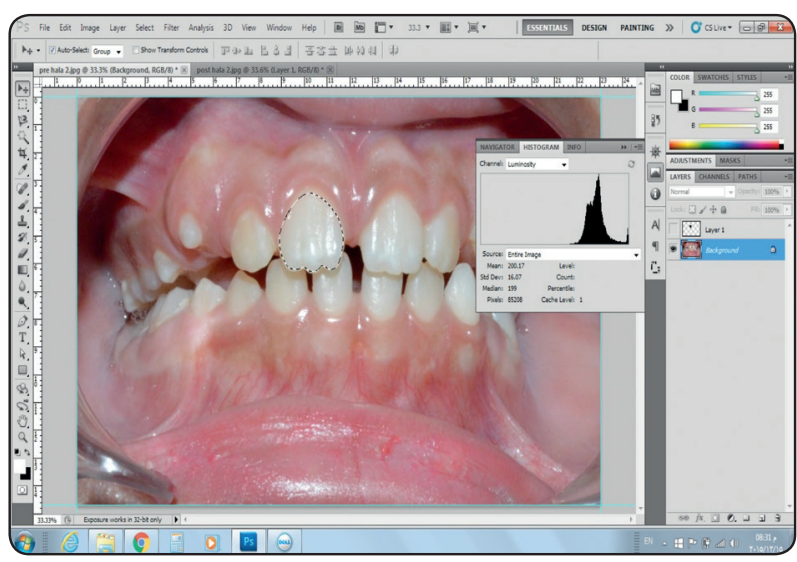

Fig. (3) Digital photography before treatment with fluoride varnish (P0)

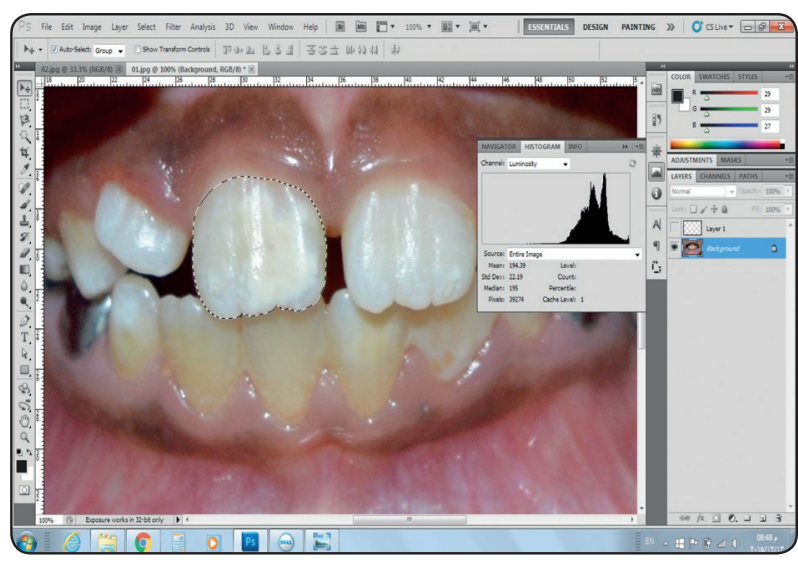

Fig. (2) Digital photography after one month treatment with resin infiltration $(\mathrm{P} 3)$

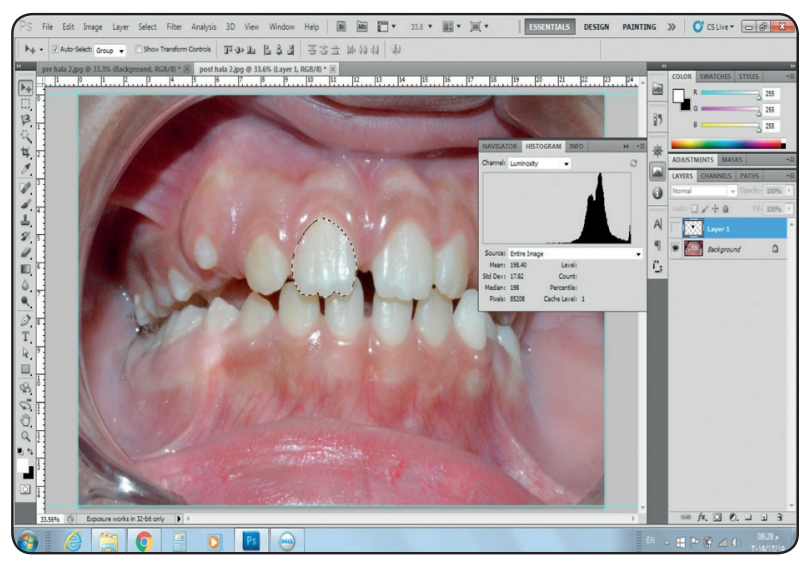

Fig. (4) Digital photography after one month treatment with fluoride varnish (P3) 


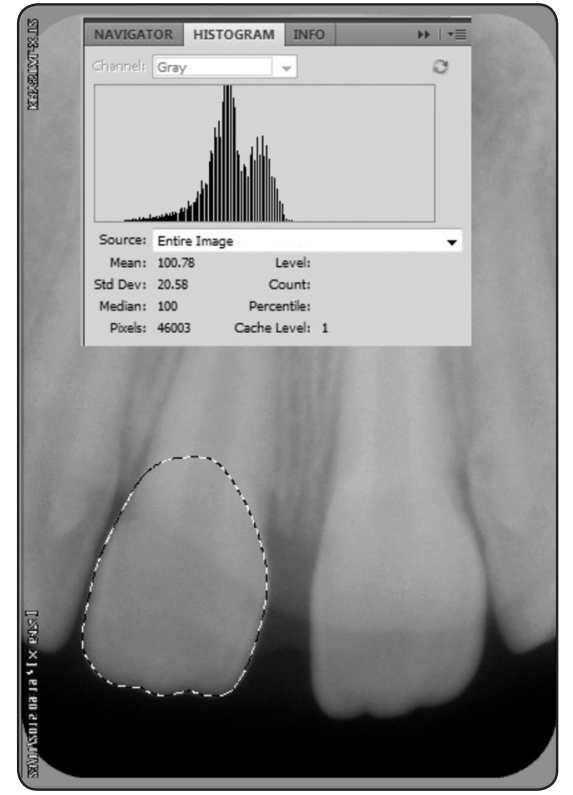

Fig. (5) Periapical radiograph analyzed by gray level system.

\section{DISCUSSION}

Hypo-mineralized incisors in MIH may present esthetic concerns to children and their parents. Micro-abrasion can be an effective treatment in shallow defects, but the defects usually extend through the full enamel thickness, also an application of the opaque resin after enamel reduction can be considered a successful restoration for hypomineralized enamel ${ }^{15,16}$. In the present study noninvasive treatments (resin infiltrations and fluoride varnish) were used to improve the color defects and they were much less invasive than micro-abrasion or restoration ${ }^{17-20}$. Enamel surfaces were dried with air for adequate diagnosis and treatment before resin infiltration or fluoride varnish application as white spot lesions become more obvious when the teeth are dry because of the different refractive indices of normal enamel, water, and air ${ }^{21}$. The process of resin infiltrations was repeated two times with the icon infiltrant to decrease polymerization shrinkage of the resin and to complete occlusion of surface micro-porosity and obliteration of the hypo-mineralized surface lesions ${ }^{22}$. The result of standardized clinical photographs in the present study showed a significant decrease in the color differences between the sound and white spot enamel after application of resin infiltration. This finding agreed with Torres et al., $2011{ }^{23}$ who explained the greater efficacy of resin infiltration in masking white spot lesion of enamel due to low viscosity, acid resistance and high diffusion coefficient of resin that lead to obstruction of micro-porosities in enamel surfaces. In addition, Paris et al., $2013{ }^{24}$ proved that infiltration was an effective method in masking white spot lesions and it had visible stable effects on enamel surface color under discoloring condition. On the other hands, in group II the color difference decreased insignificantly after fluoro-protector varnish application. This result in accordance to Chin et al., $2009{ }^{25}$ who applied low dose fluoride (250 ppm) as re-mineralizing agent for 28 days and found limited improvement of white spot lesions and the change in color was instable. The result of this study showed a statistically significant lower mean color difference in group I than in group II in all evaluation periods. Previous studies showed that, (ICON) proved to be the most effective method in masking white spot lesions and less resistant to formation of new white spot lesions when compared to treatment with therapeutic fluoride solutions ${ }^{26,27}$. Furthermore the color stability of infiltrated teeth is durable ${ }^{28,29}$. These findings may be attributed to the extremely high penetration coefficient of the Icon resin that enables it to penetrate to a depth more than $0.5 \mathrm{~mm}$ into the lesion pores by capillary action phenomena. The deep penetration of the Icon resin leads to elevation of the refractive index of hypomineralized enamel from 1.33 to 1.52 which is nearly similar to sound enamel refractive index 1.62 and gives more homogenous enamel color and an excellent cosmetic result $22,30,31$.

In the present study, the gray level (GL) results showed significant improvement in means of (GL) images taken before and after resin infiltration on the other hand the results of group II showed 
insignificant improvement in (GL) before and after fluoride varnish application. This may be due to increased surface micro hardness of the surfaces treated with resin infiltration, increased resistance to further demineralization and mineral loss ${ }^{24}$. In addition, Comparison of the GL means of the two groups showed a significant lower GL means in fluoride treated group than resin treated group in all evaluation periods. It may be attributed to the complete penetration of the resin infiltrant into the lesion body of the enamel and the formation of a deep homogenous diffusion barrier inside the lesion, by replacing lost minerals with resin ${ }^{11,32}$. Based on this study's results, resin infiltration (ICON) is promising treatment modality in masking white spot enamel lesions in children with MIH.

One of the limitations of this study was the moderate sample size due to school-aged patients which are usually difficult to collaborate in studies with follow up period. Another limitation is the high cost of resin infiltration (ICON) treatment modality in relation to other approaches. Further studies with longer periods of follow up are necessary to evaluate the clinical performance and color stability of resin infiltration (ICON) technique in masking the enamel lesions in children with MIH.

\section{CONCLUSION}

Resin infiltration (ICON) is dramatically better than fluoride varnish in the masking of white spot lesions in children with (MIH). Resin infiltration technique allows a minimally invasive treatment in a single appointment making it beneficial for school aged patients.

\section{ACKNOWLEDGMENTS}

We are grateful to all children and their families who took part in this study

\section{REFERENCES}

1- Weerheijm KL, Jälevik B and Alaluusua S. Molar-incisor hypomineralisation.Caries Res 2001;35: 390-391.

2- Ghanim A, Manton D, Bailey D, Marino R and Morgan M. Risk factors in occurance of molar - incisor hypomineralization among group of Iraq children. Int $\mathbf{J}$ Paediatr Dent 2013; 23:197-184.

3- Jälevik B and Norén JG. Enamel hypomineralization of permanent first molars: a morphological study and survey of possible aetiological factors. Int J Paediatr Dent 2000;10: 278-289.

4- A review of the developmental defects of enamel index (DDE Index). Commission on Oral Health, Research \& Epidemiology. Report of an FDI Working Group. Int Dent J 1992;42:411-426.

5- Chawla N, Messer LB and Silva M. Clinical studies on molar-incisor hypomineralisation part 1: distribution and putative associations. Eur Arch Paediatr Dent 2008; 9: 180-190.

6- Lygidakis NA. Treatment modalities in children with teeth affected by molar-incisor enamel hypomineralisation (MIH): A systematic review. Eur Arch Paediatr Dent 2010;11: 65-74.

7- Willmot DR.White lesions after orthodontic treatment: does low fluoride make a difference? J Orthod 2004; 31 : 235-24.

8- Ardu S, Castioni NV, Benbachir N and Krejci I. Minimally invasive treatment of white spot enamel lesions. Quintessence Int 2007;38: 633-636.

9- Beerens M W, Van der Veen M H, van Beek H and ten Cate J M. Effects of casein phoshopeptide amorphous calcium fluoride phosphate paste on white spot lesion and dental plaque after orthodontic treatement: a 3 mounth follow up. European Journal of Oral Sciences 2010; 118:610-617.

10- Hamdan S M, El Banna M, El Zayat I and Mohsen M A. Effect of resin infiltration on white spot lesion after debonding orthodontic brackets. American Journal of Dentistry 2012; 25:3-8.

11- Paris S, Meyer-Lueckel H, Cölfen H and Kielbassa AM. Resin infiltration of artificial enamel caries lesions with experimental light curing resins. Dent Mater J 2007; 26: 582-588. 
12- Meyer-Lueckel H and Paris S. Progression of artificial enamel caries lesions after infiltration with experimental light curing resins. Caries Res 2008; 42: 117-124.

13- Eckstein A, Helms HJ and Knösel M. Camouflage effects following resin infiltration of post-orthodontic white-spot lesions in vivo: One-year follow-up. Angle Orthod. 2015; 85(3):374-380.

14- Weerheijm KL. Molar-incisor hypomineralization (MIH). Eur J Paediatr Dent 2003; 4(3):114-120.

15- Weerheijm KL. Molar incisor hypo-mineralization (MIH): Clinical presentation, aetiology, and management. Dent Update 2004; 31:9-12.

16- Reynolds EC. New modalities for a new generation: Casein phosphopeptide-amorphous calcium phosphate, a new remineralization technology. Synopses 2005; 30:1-6.

17- Malterud MI. Minimally invasive restorative dentistry: a biomimetic approach. Pract Proced Aesthet Dent 2006; 18: 409-414.

18- Meyer-Lueckel H, Paris S and Kielbassa AM. Surface layer erosion of natural caries lesions with phosphoric and hydrochloric acid gels in preparation for resin infiltration. Caries Res 2007; 41: 223-230.

19- Stahl $\mathbf{J}$ and Zandona AF. Rationale and protocol for the treatment of non-cavitated smooth surface carious lesions. Gen Dent 2007; 55: 105-111.

20- Reynolds E.C. Calcium phosphate based remineralizing system: scientific evidence. Aust. Dent J 2008; 53(3):268-273.

21- Hosey MT, Deery C and Waterhouse PJ. Pediatric cariology. London: Quintessense Essentials 2004.

22- Kim S, Kim EY, Jeong TS et al., The evaluation of resin infilteration for masking labial enamel white spot lesions . Int J Pediatric Dent 2011; 21:241-248.

23- Torres CR, Borges A B, Torres L M , Gomes I S and de Oliveira RS. Effect of caries infiltration technique and fluoride therapy on the color of masking white spot lesions Journal of Dentistry 2011; 39:202-207.

24- Paris S, Schwendicke F, Keltsch J, Drfer C and MeyerLueckel H. Masking of white spot lesions by resin infilteration in vitro. Journal of Dentistry 2013; 41:28-34.

25- Chin MY, Sandham A, Rumachik E N and Huysmans M C. Fluoide release and cariostatic potential of orthodontic adhesives with and without daily fluoride rinsing. Ameican Journal of Orthodontic and Dentofacial Orthopedics 2009; 136:547-553.

26- Kim S, Shin JH, Kim EY, Lee SY, Yoo SG. The evaluation of resin infiltration for masking labial enamel white spot lesions. Caries Res 2010; 44: 171-248.

27- Rocha Gomes Torres C, Marcondes Sarmento Torres L, Silva Gomes I, Simões de Oliveira R, Bühler Borges A. Effect of caries infiltration technique and fluoride therapy on the color masking of white spot lesions. 2010, Data on file. DMG, Hamburg, Germany.

28- Luebbers D, Spieler-Husfeld K, Staude C. In vitro color stability of infiltrated carious lesions. 2009, Data on file. DMG, Hamburg, Germany.

29- Phark JH, Duarte S. Clinical performance and color stability of infiltrated smooth surface lesions. 2010, Data on file. DMG, Hamburg. Germany.

30- Meyer-Lückel H and Paris S. Improved resin infiltration of natural caries lesions. J Dent Res 2008; 87(12):1112-1116.

31- Meyer-Lückel H and Paris S. Infiltration of natural caries lesions with experimental resins differing in penetration coefficients and ethanol addition. Caries Res 2010; 44(4):408-414.

32- Gugnani N, Pandit IK, Gupta M, Josan R. Caries infiltration of noncavitated white spot lesions: A novel approach for immediate esthetic improvement. Contemporary Clinical Dentistry 2012;3:199-202. 